\title{
Anisotropic collisions and impact circular polarization (Research Note)
}

\section{Application to the solar Halpha line}

\begin{abstract}
M. Derouich ${ }^{\star}$
Instituto de Astrofísica de Canarias, 38205 La Laguna, Tenerife, Spain

e-mail: moncef@iac.es

Received 23 August 2006 / Accepted 5 November 2006

ABSTRACT

Aims. In light of recent contradictory observational results concerning the atomic polarization of solar H I lines, our purpose is to present certain collisional effects that might contribute towards a possible explanation. In particular, we aim to draw attention to the possibility of creating the "impact circular polarization".

Methods. A general theoretical formulation of the problem of anisotropic collisions in the tensorial representation is obtained in an arbitrary symmetry of the relative velocity distribution. To try to understand this in concrete terms and estimate the effect of these collisions for creating and increasing the atomic orientation, we determine an explicit expression of the alignment-to-orientation transfer rates between two hydrogen levels in a particular case of symmetry.

Results. The anisotropic collisions could play a role in creating and increasing the atomic orientation by an alignment-to-orientation conversion mechanism (impact circular polarization). Physically, this is due to coherence transfer by anisotropic collisions. This transfer, and hence the creation of atomic circular polarization, can be achieved in different ways, which we describe in the case of the $\mathrm{H}_{\alpha}$ line. However, for given solar conditions, the alignment-to-orientation transfer rates seem to be 4 to 5 times lower than the population-to-alignment transfer rates which are basically responsible for generating the impact linear polarization.
\end{abstract}

Key words. atomic processes - line: formation - polarization - Sun: magnetic fields - Sun: prominences - Sun: flares

\section{Introduction}

Symmetry-breaking processes, such as anisotropic illumination and anisotropic collisions, could create the so-called atomic polarization. This polarization reflects the fact that the Zeeman sublevels $(j M)$ of the atomic system are unevenly populated and characterized by definite coherence factors. In an aligned atomic system, states of different $|M|$ are unequally populated, while the populations in $M$ and $-M$ could be the same. In contrast, an orientated system is characterized by different populations in the $M$ and $-M$ states

The internal organization of the atomic system is described by the density matrix elements ${ }^{j} \rho_{q}^{k}(0 \leq k \leq 2 j$ and $-k \leq q \leq k)$ expressed on the basis of the irreducible tensorial operators $T_{q}^{k}$, which has been shown to be the most suitable to formulating the problem of the formation of polarized spectral lines (e.g. Sahal-Bréchot 1977; Landi Degl'Innocenti \& Landolfi 2004). The orientation of the atomic system is quantified by the density matrix elements with odd rank $k\left({ }^{j} \rho_{q}^{k=1},{ }^{j} \rho_{q}^{k=3}\right.$, etc. $)$, while the alignment is associated with the even ones: ${ }^{j} \rho_{q}^{k=2},{ }^{j} \rho_{q}^{k=4}$, etc. The population of the $j$-level is given by ${ }^{j} \rho_{0}^{0}$.

In the present work, we draw attention to the effect of anisotropic collisions on the density matrix components. This effect is characterized by mutual conversion of rank $k$. If

* Also associated researcher at CNRS UMR 8112 - LERMA, Observatoire de Paris, Section de Meudon, 92195 Meudon, France. anisotropic collisions are efficient under solar conditions, the population-to-alignment transfer (alignment creation), which is basically the origin of the well-known impact linear polarization, could be accompanied by alignment-to-orientaion transfer. This transfer makes the creation of the orientation of the levels by anisotropic collisions possible, leading to the observation of a symmetric Stokes $V$ parameter. We propose to call this phenomenon impact circular polarization by analogy with the impact linear polarization.

\section{Anisotropic collisional rates in the irreducible basis}

In the framework of the impact approximation, the total effect of the collisions is obtained by multiplying the collisional rate for a binary collision by the perturber density. Let us consider a binary collision between a neutral hydrogen atom and a proton. The transition matrix $T(\boldsymbol{b}, \boldsymbol{v})=I-S(\boldsymbol{b}, \boldsymbol{v})$ gives the time evolution of the state of the hydrogen atom, where $S$ is the scattering matrix, and $\boldsymbol{b}$ and $\boldsymbol{v}$ are the impact parameter and relative velocity, respectively. We notice that we are formulating the problem in a semiclassical description, but our conclusions on the tensorial component mixing of the collisional rates are independent of this approximation and are the same in a quantum approach. We use the atomic frame where the emitting atom is fixed at the origin and the quantization axis is taken as perpendicular to the collision plane $(\boldsymbol{b}, \boldsymbol{v})$. 
In the description of the hydrogen atom, we neglect the contribution of the hyperfine structure (HFS). There are no additional conceptual difficulties for including the contribution of the HFS, so the equations presented below can be obtained when the HFS is taken into account simply by carrying out some formal substitutions between fine and hyperfine quantum numbers. However, our main conclusions remain unchanged.

The probability of the transfer of the coherence from $\left|j_{2} v_{2}\right\rangle$ $\left\langle j_{2} v_{2}^{\prime}\right|$ to $\left|j_{1} \mu_{1}\right\rangle\left\langle j_{1} \mu_{1}^{\prime}\right|$ by collisions is ${ }^{1}$

$$
\begin{aligned}
& \Pi\left(j_{1} \mu_{1} \mu_{1}^{\prime} \leftarrow j_{2} v_{2} v_{2}^{\prime} ; \boldsymbol{b}, \boldsymbol{v}\right)= \\
& T\left(j_{1} \mu_{1} \leftarrow j_{2} v_{2} ; \boldsymbol{b}, \boldsymbol{v}\right) \times T\left(j_{1} \mu_{1}^{\prime} \leftarrow j_{2} v_{2}^{\prime} ; \boldsymbol{b}, \boldsymbol{v}\right)^{*}
\end{aligned}
$$

where $\mu_{1} \neq \mu_{1}^{\prime}$ and/or $v_{2} \neq v_{2}^{\prime}$. The transition probability corresponds to $\mu_{1}=\mu_{1}^{\prime}$ and $v_{2}=v_{2}^{\prime}$ :

$\Pi\left(j_{1} \mu_{1} \mu_{1} \leftarrow j_{2} v_{2} v_{2} ; \boldsymbol{b}, \boldsymbol{v}\right)=\left|T\left(j_{1} \mu_{1} \leftarrow j_{2} v_{2} ; \boldsymbol{b}, \boldsymbol{v}\right)\right|^{2}$.

In an arbitrary frame obtained in the rotation $\mathcal{D}(\alpha, \beta, \gamma)$ of the atomic frame by the Euler angles $\alpha, \beta$, and $\gamma$, the coherence transfer probability is

$$
\begin{gathered}
\Pi\left(j_{1} M_{1} M_{1}^{\prime} \leftarrow j_{2} M_{2} M_{2}^{\prime} ; \boldsymbol{b}, \boldsymbol{v}\right)=\sum_{\mu_{1}, \mu_{1}^{\prime}, v_{2}, v_{2}^{\prime}} \Pi\left(j_{1} \mu_{1} \mu_{1}^{\prime} \leftarrow j_{2} v_{2}^{\prime} v_{2}^{\prime} ; \boldsymbol{b}, \boldsymbol{v}\right) \\
\times\left[\mathcal{D}_{v_{2} M_{2}}^{\left(j_{2}\right)}\right]^{*} \mathcal{D}_{\mu_{1} M_{1}}^{\left(j_{1}\right)} \mathcal{D}_{v_{2}^{\prime} M_{2}^{\prime}}^{\left(j_{2}\right)}\left[\mathcal{D}_{\mu_{1}^{\prime} M_{1}^{\prime}}^{\left(j_{1}\right)}\right]^{*}
\end{gathered}
$$

The collisional rates associated with the transfer of the coherence from $\left|j_{2} M_{2}\right\rangle\left\langle j_{2} M_{2}^{\prime}\right|$ to $\left|j_{1} M_{1}\right\rangle\left\langle j_{1} M_{1}^{\prime}\right|$ in anisotropic collisions are

$$
\begin{array}{r}
\zeta\left(j_{1} M_{1} M_{1}^{\prime} \leftarrow j_{2} M_{2} M_{2}^{\prime}\right)=n_{\mathrm{pert}} \int v f(\boldsymbol{v}, T) \mathrm{d}^{3} \boldsymbol{v} \\
\times \int \mathrm{d}^{2} \boldsymbol{b} \Pi\left(j_{1} M_{1} M_{1}^{\prime} \leftarrow j_{2} M_{2} M_{2}^{\prime} ; \boldsymbol{b}, \boldsymbol{v}\right)
\end{array}
$$

where $f(\boldsymbol{v}, T)$ is the velocity distribution for a local temperature $T$ and $n_{\text {pert }}$ the perturber density. In the tensorial basis, one can readily show that

$$
\begin{aligned}
D_{q \leftarrow q^{\prime}}^{k \leftarrow k^{\prime}}\left(j_{1} \leftarrow j_{2}\right)=\sqrt{(2 k+1)\left(2 k^{\prime}+1\right)} \\
\quad \times \sum_{M_{1}, M_{1}^{\prime}} \sum_{M_{2}, M_{2}^{\prime}}(-1)^{j_{1}+j_{2}-M_{1}-M_{2}} \\
\quad \times\left(\begin{array}{ccc}
j_{1} & j_{1} & k \\
M_{1}-M_{1}^{\prime}-q
\end{array}\right)\left(\begin{array}{ccc}
j_{2} & j_{2} & k^{\prime} \\
M_{2}-M_{2}^{\prime}-q^{\prime}
\end{array}\right) \zeta\left(j_{1} M_{1} M_{1}^{\prime} \leftarrow j_{2} M_{2} M_{2}^{\prime}\right),
\end{aligned}
$$

implying that

$$
\begin{aligned}
& D_{q \leftarrow q^{\prime}}^{k \leftarrow k^{\prime}}\left(j_{1} \leftarrow j_{2}\right)=\sqrt{(2 k+1)\left(2 k^{\prime}+1\right)} \\
& \times \sum_{M_{1}, M_{1}^{\prime}} \sum_{M_{2}, M_{2}^{\prime}}(-1)^{j_{1}+j_{2}-M_{1}-M_{2}}\left(\begin{array}{ccc}
j_{1} & j_{1} & k \\
M_{1}-M_{1}^{\prime}-q
\end{array}\right)\left(\begin{array}{ccc}
j_{2} & j_{2} & k^{\prime} \\
M_{2}-M_{2}^{\prime}-q^{\prime}
\end{array}\right) \\
& \times n_{\text {pert }} \int v f(\boldsymbol{v}, T) \mathrm{d}^{3} \boldsymbol{v} \int \mathrm{d}^{2} \boldsymbol{b} \sum_{\mu_{1}, \mu_{1}^{\prime}, v_{2}, v_{2}^{\prime}} \Pi\left(j_{1} \mu_{1} \mu_{1}^{\prime} \leftarrow j_{2} v_{2} v_{2}^{\prime} ; \boldsymbol{b}, \boldsymbol{v}\right) \\
& \times\left[\mathcal{D}_{v_{2} M_{2}}^{\left(j_{2}\right)}\right]^{*} \mathcal{D}_{\mu_{1} M_{1}}^{\left(j_{1}\right)} \mathcal{D}_{v_{2}^{\prime} M_{2}^{\prime}}^{\left(j_{2}\right)}\left[\mathcal{D}_{\mu_{1}^{\prime} M_{1}^{\prime}}^{\left(j_{1}\right)}\right]^{*}
\end{aligned}
$$

1 The left arrow $(\leftarrow)$ is used because the transfer of the coherence from $\left|j_{2} v_{2}\right\rangle\left\langle j_{2} v_{2}^{\prime}\right|$ to $\left|j_{1} \mu_{1}\right\rangle\left\langle j_{1} \mu_{1}^{\prime}\right|$ represents gain terms in the statistical equilibrium equations describing the evolution of the $j_{1}$-level.
Using the contraction properties of the rotations matrix and after some algebraic transformations one can find ${ }^{2}$

$$
\begin{aligned}
& D_{q \leftarrow q^{\prime}}^{k \leftarrow k^{\prime}}\left(j_{1} \leftarrow j_{2}\right)=(2 k+1)\left(2 k^{\prime}+1\right) \\
& \times \sum_{\mu_{1}, \mu_{1}^{\prime}, v_{2}, v_{2}^{\prime}} \sum_{L, m_{L}, m_{L}^{\prime}} \sum_{P, P^{\prime}}(2 L+1)(-1)^{j_{1}+j_{2}-\mu_{1}-v_{2}+q^{\prime}-P^{\prime}} \\
& \times\left(\begin{array}{ccc}
j_{1} & j_{1} & k \\
\mu_{1}^{\prime}-\mu_{1} P
\end{array}\right)\left(\begin{array}{ccc}
k & k^{\prime} & L \\
q-q^{\prime} & m_{L}
\end{array}\right)\left(\begin{array}{ccc}
k & k^{\prime} & L \\
P & P^{\prime} & m_{L}^{\prime}
\end{array}\right)\left(\begin{array}{ccc}
j_{2} & j_{2} & k^{\prime} \\
v_{2}-v_{2}^{\prime} P^{\prime}
\end{array}\right) n_{\text {pert }} \int v f(\boldsymbol{v}, T) \mathrm{d}^{3} \boldsymbol{v} \\
& \times \int \mathrm{d}^{2} \boldsymbol{b} \Pi\left(j_{1} \mu_{1} \mu_{1}^{\prime} \leftarrow j_{2} v_{2} v_{2}^{\prime} ; \boldsymbol{b}, \boldsymbol{v}\right) \times\left[\mathcal{D}_{m_{L}^{\prime} m_{L}}^{(L)}\right] \text {. }
\end{aligned}
$$

Accurate calculation of the $D_{q \leftarrow q^{\prime}}^{k \leftarrow k^{\prime}}\left(j_{1} \leftarrow j_{2}\right)$ depends mainly on determing of the velocity distribution $f(v, T)$ and the probability of the transfer of the coherence $\Pi\left(j_{1} \mu_{1} \mu_{1}^{\prime} \leftarrow j_{2} v_{2} v_{2}^{\prime} ; \boldsymbol{b}, \boldsymbol{v}\right)$. The velocity distribution $f(v, T)$ depends on the medium in which the collision occurs. The value of $\Pi\left(j_{1} \mu_{1} \mu_{1}^{\prime} \leftarrow j_{2} v_{2} v_{2}^{\prime} ; \boldsymbol{b}, \boldsymbol{v}\right)$ should be obtained after solving the Schrödinger equation, which implies that the interaction potential has to be calculated.

From the general formula (7) one can recover the limiting case of isotropic collisions. In fact, in isotropic symmetry, the effect of the collisions is the same for diagonal density matrix elements corresponding to populations where $\mu_{1}=\mu_{1}^{\prime}$ and $\nu_{2}=$ $v_{2}^{\prime}$ or for off-diagonal ones corresponding to coherences where $\mu_{1} \neq \mu_{1}^{\prime}$ and/or $v_{2} \neq v_{2}^{\prime}$. In addition, one retains only the $L=$ 0 term, which corresponds to an isotropic part of the velocity distribution. We then obtain

$$
\begin{aligned}
D^{k}\left(j_{1} \leftarrow j_{2}\right)= & (2 k+1) \sum_{\mu_{1}, v_{2}}(-1)^{j_{1}+j_{2}-\mu_{1}-v_{2}} \\
& \times\left(\begin{array}{ccc}
j_{1} & j_{1} & k \\
\mu_{1} & -\mu_{1} & 0
\end{array}\right)\left(\begin{array}{ccc}
j_{2} & j_{2} & k \\
v_{2} & -v_{2} & 0
\end{array}\right) \zeta\left(j_{1} \mu_{1} \leftarrow j_{2} v_{2}\right)
\end{aligned}
$$

This expression has been obtained by Sahal-Bréchot (1977) (see also Follmeg et al. 1990) in another way and used in our previous calculations concerned with isotropic collisions (e.g. Eq. (3) of Derouich et al. 2003). It is useful to note that for isotropic processes the coupling terms implying $k \neq k^{\prime}$ and transfer of coherence $q$ to $q^{\prime}$ are zero, and that the depolarization and polarization transfer rates are $q$-independent.

Equation (7) demonstrates the theoretical possibility of mixing the tensorial orders $k$ if the collisions are anisotropic. The question now is how this mixing depends on the symmetry of the problem.

\section{Collisional effect in the particular case of cylindrical symmetry}

Consider an atomic system in an anisotropic environment having cylindrical symmetry around a given direction that we choose as the quantization axis of total angular momentum (the $z$-axis of our reference system). We imagine the anisotropic collisions to be a superposition of directive collisions organized in cylindrical symmetry around the anisotropy $z$-axis. If one defines the purely directive probability of the coherence transfer in the tensorial basis as

$$
\begin{aligned}
& \Pi_{P}^{k \leftarrow k^{\prime}}\left(j_{1} \leftarrow j_{2} \text {, directive }\right)=\sum_{\mu_{1}, \mu_{1}^{\prime}, v_{2}, v_{2}^{\prime}}(-1)^{j_{1}+j_{2}-\mu_{1}-v_{2}}
\end{aligned}
$$

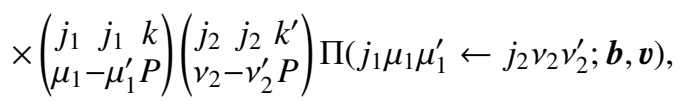

\footnotetext{
${ }^{2}$ Details of the demonstration of the Eq. (7) are available on request from the author.
} 
after some appropriate transformations, we find that

$$
\begin{aligned}
& D^{k \leftarrow k^{\prime}}\left(j_{1} \leftarrow j_{2}, \text { cylindric }\right)=(2 k+1)\left(2 k^{\prime}+1\right)
\end{aligned}
$$

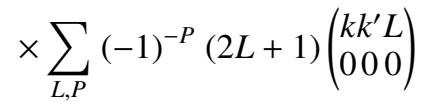

$$
\begin{aligned}
& \times\left(\begin{array}{lll}
k & k^{\prime} & L \\
P & -P 0
\end{array}\right) \times D_{P}^{k \leftarrow k^{\prime}}\left(j_{1} \leftarrow j_{2}, \text { directive }\right)
\end{aligned}
$$

where $D_{P}^{k \leftarrow k^{\prime}}\left(j_{1} \leftarrow j_{2}\right.$, directive $)$ is the purely directive polarization transfer for a given plane of collision $(\boldsymbol{v}, \boldsymbol{b})$, i.e. obtained by integrating $\Pi_{P}^{k \leftarrow k^{\prime}}\left(j_{1} \leftarrow j_{2}\right.$, directive) over the velocities and impact parameters in a given direction of the plane of the collisions before taking any average over the angles. Or for a cylindrical symmetry $^{3}$,

$f(v, \theta, T)=f(v,-\theta+\pi, T)$.

Using this property of the Legendre polynomials

$P_{L}(\theta)=P_{L}(-\theta+\pi) \quad$ if $L$ is even and

$P_{L}(\theta)=-P_{L}(-\theta+\pi)$ if $L$ is odd,

one concludes that $L$ is necessarily even. But also

$\left(\begin{array}{ccc}k & k^{\prime} & L \\ 0 & 0 & 0\end{array}\right) \neq 0$, only if $k+k^{\prime}+L$ is even.

Equations (12) and (13) mean that the sum $k+k^{\prime}$ is necessarily even. Linear polarization can be produced by anisotropic cylindrical excitation of the emitting atoms mainly due to the population $(k=0)$-to-alignment $(k=2)$ transfer. The well-known example in astrophysics is the observation of the linear polarization in the $\mathrm{H}_{\alpha}$ line in solar flares (e.g. Hénoux et al. 1990), which is interpreted as due to anisotropic collisions between hydrogen atoms and background charged particles - the impact of accelerated beams of electrons and/or protons on hydrogen atoms. However, rates with $k+k^{\prime}$ odd vanish, leading to the impossibility of collisional transfer of the atomic alignment to orientation, or in other words, the impossibility of the collisional emergence of circular polarization from the existing atomic linear polarization.

We mention that there is now international controversy over the existence of impact linear polarization during solar flares (Bianda et al. 2005). Here we assume that such an impact linear polarization exists and demonstrate the theoretical possibility of impact circular polarization in the case of cylindrical symmetry breaking.

\section{Cylindrical symmetry-breaking: the emergence of impact circular polarization}

Let us consider now an atomic system illuminated by linearly polarized or unpolarized light having cylindrical symmetry around a $z$-axis. The alignment-to-orientation conversion may be induced by anisotropic collisions having a cylindrical symmetry around the anisotropy axis if the angle between the anisotropy axis and that of $z$-axis differs from 0 or $\frac{\pi}{2}$ (Lombardi 1967; Rebane 1968). Then, the breaking of the cylindrical symmetry of the problem can cause alignment to orientation conversion. This physical situation can occur when charged plasma particles

3 It should be noted that the cylindrical symmetry alone do not allows Eq. (11). That equation becomes correct thanks to time reversal invariance - the perturbed atom is insensitive to the sense of the movement of the beams of the perturbers. (e.g. protons or electrons) move along magnetic field having a direction different from that of the radiation field.

To understand the effect of the anisotropic collisions in concrete terms, we assume that these collisions, apart of the cylindrical symmetry around the anisotropy axis, they have a symmetry of reflection with respect to planes passing through this axis. Therefore, $D_{q \leftarrow q^{\prime}}^{k \leftarrow k^{\prime}}\left(j_{1} \leftarrow j_{2}\right)$ vanishes if $q \neq q^{\prime}$, but unlike the case of Sect. 3 where the problem has a cylindrical symmetry, one has $q \neq 0$. Although this is a particular case of symmetry, our conclusions about the possibility of the transfer of the alignment to orientation are applicable for any anisotropic non-cylindrical distribution.

The expression of $D_{q}^{k \leftarrow k^{\prime}}\left(j_{1} \leftarrow j_{2}\right)$ could be obtained from Eq. (7):

$$
\begin{aligned}
& D_{q}^{k \leftarrow k^{\prime}}\left(j_{1} \leftarrow j_{2}\right)=(2 k+1)\left(2 k^{\prime}+1\right) \sum_{L, P}(-1)^{q-P} \\
& \quad \times(2 L+1)\left(\begin{array}{cc}
k & k^{\prime} L \\
q-q 0
\end{array}\right)\left(\begin{array}{ccc}
k & k^{\prime} & L \\
P-P 0
\end{array}\right) D_{P}^{k \leftarrow k^{\prime}}\left(j_{1} \leftarrow j_{2}, \text { directive }\right) .
\end{aligned}
$$

Let us consider the $\mathrm{HI}$ electronic states $3 s$ and $3 p$ where $j_{1}=$ $1 / 2, j_{2}=3 / 2, k=1$, and $k^{\prime}=2$. We find that

$$
\begin{aligned}
& D_{1}^{1 \leftarrow 2}(1 / 2 \leftarrow 3 / 2)=n_{\text {pert }} \int v f(\boldsymbol{v}, T) \mathrm{d}^{3} \boldsymbol{v} \\
& \quad \times \int \mathrm{d}^{2} \boldsymbol{b} \times P_{2}(\cos \theta) \times \sqrt{\frac{15}{8}} \\
& \quad \times\left[-\Pi\left(j_{1} \frac{1}{2} \frac{-1}{2} \leftarrow j_{2} \frac{3}{2} \frac{1}{2}\right)-\Pi\left(j_{1} \frac{-1}{2} \frac{1}{2} \leftarrow j_{2} \frac{-3}{2} \frac{-1}{2}\right)\right. \\
& \left.+\Pi\left(j_{1} \frac{-1}{2} \frac{1}{2} \leftarrow j_{2} \frac{1}{2} \frac{3}{2}\right)+\Pi\left(j_{1} \frac{1}{2} \frac{-1}{2} \leftarrow j_{2} \frac{-1}{2} \frac{-3}{2}\right)\right] .
\end{aligned}
$$

It is worth noticing that

$$
\begin{aligned}
\Pi\left(j_{1} \mu_{1} \mu_{1}^{\prime} \leftarrow j_{2} v_{2} v_{2}^{\prime}\right)= & (-1)^{\mu_{1}-\mu_{1}^{\prime}+v_{2}-v_{2}^{\prime}} \\
& \times\left[\Pi\left(j_{1}-\mu_{1}-\mu_{1}^{\prime} \leftarrow j_{2}-v_{2}-v_{2}^{\prime}\right)\right] \\
= & {\left[\Pi\left(j_{1} \mu_{1}^{\prime} \mu_{1} \leftarrow j_{2} v_{2}^{\prime} v_{2}\right)\right]^{*} ; }
\end{aligned}
$$

then

$$
\begin{aligned}
& \Pi\left(j_{1} \frac{1}{2} \frac{-1}{2} \leftarrow j_{2} \frac{-1}{2} \frac{-3}{2}\right)-\Pi\left(j_{1} \frac{-1}{2} \frac{1}{2} \leftarrow j_{2} \frac{-3}{2} \frac{-1}{2}\right)= \\
& 2 \mathrm{i} \times \operatorname{Im}\left(\Pi\left(j_{1} \frac{1}{2} \frac{-1}{2} \leftarrow j_{2} \frac{-1}{2} \frac{-3}{2}\right)\right)
\end{aligned}
$$

$\Pi\left(j_{1} \frac{-1}{2} \frac{1}{2} \leftarrow j_{2} \frac{1}{2} \frac{3}{2}\right)-\Pi\left(j_{1} \frac{1}{2} \frac{-1}{2} \leftarrow j_{2} \frac{3}{2} \frac{1}{2}\right)=$

$$
2 \mathrm{i} \times \operatorname{Im}\left(\Pi\left(j_{1} \frac{-1}{2} \frac{1}{2} \leftarrow j_{2} \frac{1}{2} \frac{3}{2}\right)\right)
$$

$\Pi\left(j_{1} \frac{-1}{2} \frac{1}{2} \leftarrow j_{2} \frac{1}{2} \frac{3}{2}\right)=\Pi\left(j_{1} \frac{1}{2} \frac{-1}{2} \leftarrow j_{2} \frac{-1}{2} \frac{-3}{2}\right)$,

so that

$$
\begin{aligned}
& D_{1}^{1 \leftarrow 2}\left(\frac{1}{2} \leftarrow \frac{3}{2}\right)=n_{\text {pert }} \times \int v f(\boldsymbol{v}, T) \mathrm{d}^{3} \boldsymbol{v} \int \mathrm{d}^{2} \boldsymbol{b} \\
& \quad \times P_{2}(\cos \theta) \sqrt{30} \mathrm{i} \times \operatorname{Im}\left(\Pi\left(j_{1} \frac{-1}{2} \frac{1}{2} \leftarrow j_{2} \frac{1}{2} \frac{3}{2}\right)\right)
\end{aligned}
$$


which means in particular that the alignment-to-orientation conversion is due to the transfer of coherence as from $\left|j_{2} \frac{1}{2}\right\rangle\left\langle j_{2} \frac{3}{2}\right|$ to $\left|j_{1} \frac{-1}{2}\right\rangle\left\langle j_{1} \frac{1}{2}\right|$, and that $D_{1}^{1 \leftarrow 2}(1 / 2 \leftarrow 3 / 2)$ is purely imaginary. This last property can also be immediately retrieved from the properties of $D_{q}^{k \leftarrow k^{\prime}}\left(j_{1} \leftarrow j_{2}\right)$ in the tensorial representation:

$$
\begin{aligned}
& D_{q}^{k \leftarrow k^{\prime}}\left(j_{1} \leftarrow j_{2}\right)=\left[D_{-q}^{k \leftarrow k^{\prime}}\right]^{*}\left(j_{1} \leftarrow j_{2}\right) \\
& \text { and } D_{q}^{k \leftarrow k^{\prime}}\left(j_{1} \leftarrow j_{2}\right)=(-1)^{k+k^{\prime}} D_{-q}^{k \leftarrow k^{\prime}}\left(j_{1} \leftarrow j_{2}\right) .
\end{aligned}
$$

We stress that $D_{q}^{k \leftarrow k^{\prime}}\left(j_{1} \leftarrow j_{2}\right)$ is purely imaginary when $k+k^{\prime}$ is odd because we considered a particular case of symmetry. But the alignment $\left(k^{\prime}=2\right)$-to-orientation $(k=1)$ transfer rate is generally complex. This rate is manifested by the conversion of the alignment components ${ }^{j_{2}} \rho_{ \pm 1}^{2}$ of the $j_{2}$-level into orientation inside the level $j_{1}$ represented by ${ }^{j_{1}} \rho_{ \pm 1}^{1}$. Alignment-to-orientation transfer effects have been reported in numerous papers; see, for example, Petrashen' et al. (1993), which also contains extensive references.

As in the case of impact linear polarization, circular polarization can be generated from bombardment by background charged particles having an anisotropic velocity distribution, so we propose to call it impact circular polarization. It is interesting to note that this circular impact polarization is possible only for anisotropic non-cylindrical velocity distribution. In contrast, impact linear polarization can be created even if the velocity distribution is cylindrical.

The alignment-to-orientation transfer rates have been calculated theoretically within the limit of a dipole-dipole interaction by Manabe et al. (1979); the same authors in 1981 gave first experimental evidence of transfer from alignmentto-orientation (Manabe et al. 1981). They found in particular that the diagonal elements of the depolarization $D$-matrix $D_{q}^{k \leftarrow k}$ are about 5 to 10 times higher than the alignmentto-orientation transfer rates $D_{q \neq 0}^{k \leftarrow k^{\prime}}$ (off-diagonal components with $k \neq k^{\prime}$ ), depending on the degree of anisotropy of the velocity distribution. Of course, the usual rates $D_{q=0}^{k \leftarrow k}$, which are non-zero even for isotropic collisions, are especially higher than $D_{q}^{k \leftarrow k+1}$. This is because they first depend on the transition probabilities $\Pi\left(j_{1} \mu_{1} \mu_{1} \leftarrow j_{2} v_{2} v_{2} ; \boldsymbol{b}, \boldsymbol{v}\right)$, but $D_{q}^{k \leftarrow k+1}$ depends on the collisional coherence probabilities $\Pi\left(j_{1} \mu_{1} \mu_{1}^{\prime} \leftarrow j_{2} v_{2} v_{2}^{\prime} ; \boldsymbol{b}, \boldsymbol{v}\right)\left(\mu_{1} \neq \mu_{1}^{\prime}\right.$ and $\left.\nu_{2} \neq v_{2}^{\prime}\right)$, which are typically lower. Second, $D_{0}^{k \leftarrow k}$ is generated by the isotropic and anisotropic parts of the velocity distribution, whereas $D_{q \neq 0}^{k \leftarrow k+1}$ is only created by the anisotropic part where $L \neq 0$.

The rate $D_{1}^{1 \leftarrow 2}\left(j_{1} \leftarrow j_{2}\right)$ should be lower than $D_{q=0}^{2 \leftarrow 0}\left(j_{1} \rightarrow j_{2}\right)$, in spite of the fact that both result from the anisotropic part of the velocity distribution alone. This is mainly because $D_{q=0}^{2 \leftarrow 0}\left(j_{1} \rightarrow j_{2}\right)$ depends on the transition probabilities $\Pi\left(j_{1} \mu_{1} \mu_{1} \rightarrow j_{2} v_{2} v_{2} ; \boldsymbol{b}, \boldsymbol{v}\right)$, but $D_{1}^{1 \leftarrow 2}\left(j_{1} \leftarrow j_{2}\right)$ depends on the collisional coherence probabilities $\Pi\left(j_{1} \mu_{1} \mu_{1}^{\prime} \leftarrow j_{2} v_{2} v_{2}^{\prime} ; \boldsymbol{b}, \boldsymbol{v}\right)\left(\mu_{1} \neq \mu_{1}^{\prime}\right.$ and $\left.v_{2} \neq v_{2}^{\prime}\right)$. We expect a factor 4 to 5 between alignment-to-orientation and populationto-alignment transfer rates ${ }^{4}$. As a consequence, the impact circular polarization degree would be smaller than the impact

\footnotetext{
4 A similar conclusion was reached when we calculated the tensorial components $\zeta_{q=0}^{k=0}(j)$ and $\zeta_{q=0}^{k=1}(j)$ describing the elastic scattering and the depolarizing rates $D_{q=0}^{k=1}(j)$. In fact, $\zeta_{q=0}^{k=0}(j)$ and $\zeta_{q=0}^{k=1}(j)$ are sensitive to the diagonal elements of the $T(\boldsymbol{b}, \boldsymbol{v})$-matrix and are about $\sim 4$ to 5 times higher than $D_{q=0}^{k=1}(j)$, which depends only on the off-diagonal elements (see Eqs. (15) and (16) of Derouich \& Barklem 2007).
}

linear polarization. This might partially explain why the observed Stokes $V$ is customarily anti-symmetric, i.e. a footprint of the Zeeman effect, and the impact polarization is believed to be only linear.

We note that, because of the particular case of symmetry adopted here, $D_{q=0}^{1 \leftarrow 2}(1 / 2 \leftarrow 3 / 2)=0$, as can be easily seen from Eq. (19). If this particular case of symmetry breaks down, $D_{q=0}^{1 \leftarrow 2}(1 / 2 \leftarrow 3 / 2)$ becomes non-zero and $D_{1}^{1 \leftarrow 2}(1 / 2 \leftarrow 3 / 2)$ increases. We notice that the appearance of the non-diagonal rates $D_{q \leftarrow q^{\prime}}^{k \leftarrow k^{\prime}}(1 / 2 \leftarrow 3 / 2)$ is completely due to the anisotropy of the relative velocity distribution, but that their increase is mainly due to the value of the relative velocity rather than the anisotropy (Manabe et al. 1979).

\section{Implications in solar physics}

The calculation of the collisional transfer probabilities $\Pi\left(j_{1} \mu_{1} \mu_{1}^{\prime} \leftarrow j_{2} v_{2} v_{2}^{\prime}\right)$ requires, first, the calculation of the required atomic wavefunctions and interaction potentials and, second, the resolution of the time-dependent Schrödinger equation. In the collisional semi-classical method of Sahal-Bréchot et al. (1996), only the probabilities of transition where $\mu_{1}=\mu_{1}^{\prime}$ and $v_{2}=v_{2}^{\prime}$ were taken into account since they have been concerned with the impact linear polarization of the $\mathrm{H}_{\alpha}$ line in solar flares. Sahal-Bréchot et al. (1996) have obtained the transition probability by using the formulae of Seaton (1962), i.e. without solving the Schrödinger equation. We think that a careful study based on accurate interaction potentials and a close coupling dynamical description is highly desirable to try to improve the calculations of Sahal-Bréchot et al. (1996), where $\mu_{1}=\mu_{1}^{\prime}$ and $v_{2}=v_{2}^{\prime}$, and most importantly to calculate the coherence probabilities where $\mu_{1} \neq \mu_{1}^{\prime}$ and $v_{2} \neq v_{2}^{\prime}$.

The $n \mathrm{~s}, n \mathrm{p}$, and $n$ d levels are remarkably close in the case of hydrogen levels, for example, $E\left(2 \mathrm{p}{ }^{2} \mathrm{P}_{3 / 2}\right)-E\left(2 \mathrm{~s}{ }^{2} \mathrm{~S}_{1 / 2}\right)=$ $0.33 \mathrm{~cm}^{-1}$ and $E\left(3 \mathrm{p}^{2} \mathrm{P}_{3 / 2}\right)-E\left(3 \mathrm{~s}^{2} \mathrm{~S}_{1 / 2}\right)=0.098 \mathrm{~cm}^{-1}$, making the collisional coherence transfer between different electronic states more efficient and consequently the emergence of impact circular polarization. Moreover, it could be important to take the coherence transfer inside the same electronic state into account. The efficiency of the different possibilities of the transfer of the coherence depends of the energy of the perturbers.

If the cylindrical symmetry breaks, anisotropic collisions could convert alignment into orientation independently on the values of the magnetic and electric fields. The regime of $j$-level crossing is not needed for the collisional alignment-toorientation transfer. It is might be of interest to recall that, unlike electric dipole radiative transitions, collisional processes do not obey strong selection rules.

The seven components of the $\mathrm{H}_{\alpha}$ line connect the hydrogen electronic state $3 p$ to $2 s$, the 3 s to $2 p$, and the $3 d$ state to $2 p$. The observation of circular polarization (López Ariste et al. 2005) attributed to the orientation of one of these levels could be due to the coherence transfer by anisotropic collisions. For instance (see Fig. 1):

- the relatively long-lived metastable level $2 s{ }^{2} S_{1 / 2}$ is particularly exposed to the effect of coherence transfer by anisotropic collisions; this level is circularly polarizable by alignment-to-orientation transfer processes with the level $2 \mathrm{p}^{2} \mathrm{P}_{3 / 2}$;

- the level $3 \mathrm{~s}{ }^{2} \mathrm{~S}_{1 / 2}$ can become orientated by alignment-toorientation transfer processes with the level $3 \mathrm{p}^{2} \mathrm{P}_{3 / 2}$;

- the upper level of one component of the $\mathrm{H}_{\alpha}$ line $3 \mathrm{p}{ }^{2} \mathrm{P}_{3 / 2}$ can be aligned as a result of the anisotropic collisions or 


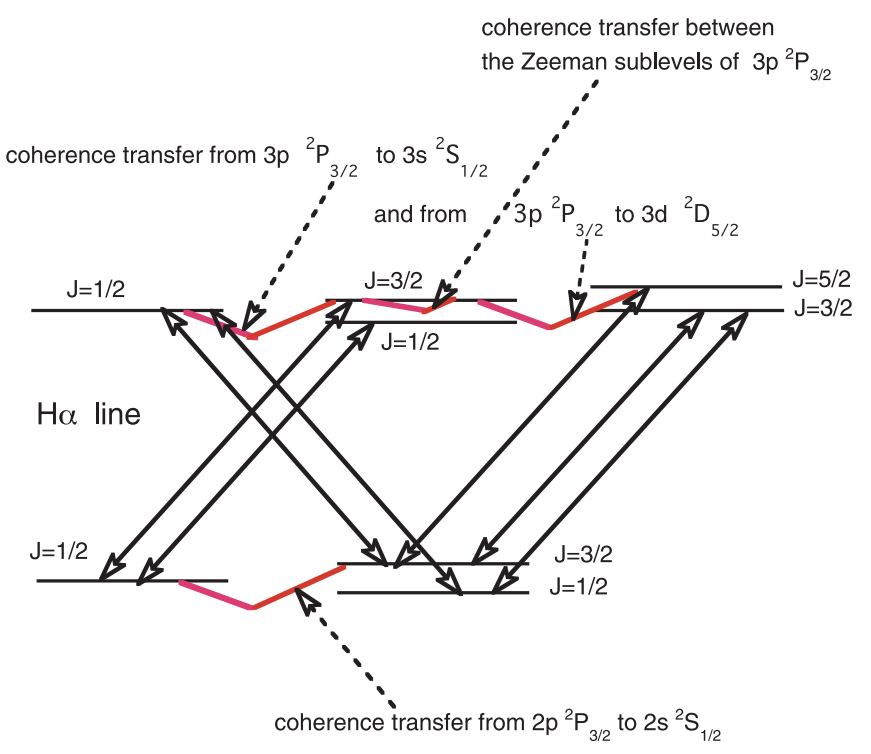

$J=1 / 2$

Fig. 1. Schematic representation of non-exclusive ways for the collisional transfer of coherence between different electronic states or in the same state. As a result, impact circular polarization of the emitted light could be observed. The states of the hydrogen atom taken into account are $1 \mathrm{~s}, 2 \mathrm{~s}, 2 \mathrm{p}, 3 \mathrm{~s}, 3 \mathrm{p}$, and $3 \mathrm{~d}$. Note that the level spacings are not to scale.

radiation; this alignment can convert to orientation of the $3 p^{2} \mathrm{P}_{3 / 2}$, leading to circular impact polarization. In this case, expressions of the corresponding transfer rates associated with this process can be obtained from the above general equations by taking $j_{1}=j_{2}=3 / 2$.

This reasoning is applicable to other levels intervening in the $\mathrm{H}_{\alpha}$ line structure. It can also be applied to other atomic lines.

It is useful to note that, since the hyperfine levels are closer than fine levels, the coherence transfer probabilities between hyperfine levels may be important. Collisional alignment-toorientation transfer rates may then play a role for atoms or ions with important hyperfine-structure effects.

\section{Conclusion}

Spectropolarimetric observations by López Ariste et al. (2005) of the $\mathrm{H}_{\alpha}$ line in solar prominences show unexpected atomic orientation $(k=1)$ in the hydrogen levels. On the other hand, Ramelli et al. (2005) have recently found Stokes $V$ profiles showing only the typical 2-lobe antisymmetric pattern profiles due to the Zeeman effect. Our aim was to pose the problem from a collisional point of view.
Theoretical investigation of the effect of anisotropic collisions shows the possibility of impact circular polarization, i.e. the creation of symmetric Stokes $V$ by anisotropic noncylindrical collisions. Physically, the collisional transfer of coherence between hydrogen levels is responsible for this creation, which reflects the transfer of the alignment of a $j_{2}$-level ${ }^{j_{2}} \rho_{ \pm 1}^{2}$ to the orientation of a $j_{1}$-level represented by ${ }^{j_{1}} \rho_{+1}^{1}$. For a given solar conditions, the rate of the creation of circular atomic polarization (symmetric Stokes $V$ ) seems to be 4 to 5 times lower than that responsible for the creation of the well known impact linear polarization.

Anisotropic collisions can be efficient in the generation of symmetric Stokes $V$ (atomic orientation) if the anisotropy axis have a direction different from that of the radiation field. The cylindrical symmetry of the problem could be broken for example by the presence of a magnetic field having a direction different from that of the light and guiding the beams of the perturbers. Anisotropic collisions could convert alignment into orientation regardless if the magnetic regime of level crossing is attempt or not.

To complement this study, accurate calculations of all the collisional rates intervening in the formation of hydrogen lines, taking coherence transfer into account, are highly desirable. These collisional rates, which enter the coupled set of equations of radiative transfer and statistical equilibrium, would be important for improving our understanding of the solar prominences and flares.

Acknowledgements. Partial support by the Spanish Ministerio de Educatión y Ciencia through project AYA2004-05792 and by the European Solar Magnetism Network is gratefully acknowledged. I thank Paul Barklem for his careful reading of the manuscript and for his scientific comments that led to its improvement. I gratefully acknowledge Sylvie Sahal-Bréchot for fruitful discussions and encouragement.

\section{References}

Bianda, M., Benz, A. O., Stenflo, J. O., Küveler, G., \& Ramelli, R. 2005, A\&A, 434,1183

Derouich, M., Sahal-Bréchot, S., \& Barklem, P. S. 2003, A\&A, 409, 369

Derouich, M., \& Barklem, P. S. 2007, A\&A, 462, 1171

Follmeg, B., Rosmus, P., \& Werner, H.-J. 1990 , J. Chem. Phys., 93, 4687

Hénoux, J.-C., \& Chambe, G. 1990, J. Quant. Spectr. Radiat. Transf., 44, 193

Manabe, T., Yabuzaki, T., \&, Ogawa, T. 1979, Phys. Rev. A, 20, 1946

Manabe, T., Yabuzaki, T., \&, Ogawa, T. 1981, Phys. Rev. Lett., 46, 637

Landi Degl'Innocenti, E., \& Landolfi, M. 2004, Polarization in Spectral Lines (Dordrecht: Kluwer)

Lombardi, M. 1967, C. R. Acad. Sci. B, 265, 191

López Ariste, A., Casini, R., Paletou, F., et al. 2005, ApJ, 621, L145

Ramelli, R., Bianda, M., Trujillo Bueno, J., Merenda, L., \& Stenflo, J. O. 2005,

Proc. International Scientific Conference on Chromospheric and Coronal Magnetic Fields, ESA SP-596, ed. D. E. Innes, A. Lagg, \& S. K. Solanki, Published on CDROM, 82.1

Rebane, V. N. 1968, Opt. Spectr. USSR, 51, 163

Petrashen, A. G., Rebane, V. N., \& Rebane, T. K. 1993, JETP, Issue 2, 77, 187

Seaton, M. J. 1962, Proc. Phys. Soc., 79, 1105

Sahal-Bréchot, S. 1977, ApJ., 213, 887

Sahal-Bréchot, S., Vogt, E., Thoraval, S., \& Diedhiou, I. 1996, A\&A, 309, 317, 